\title{
Radio-dating the Galaxy
}

\section{Gerry Gilmore}

THE determination of the age of the Universe is one of the most important, but most difficult, problems in modern astronomy. In standard models, the age of the Universe depends primarily on the present rate of expansion (measured as the Hubble constant) and deceleration rate, which is determined by the total mass in the Universe. Thus, comparison of the Hubble age of the Universe with an independent age is a test of cosmological models. The most commonly used alternative measure of age is derived from the oldest stars known, using stellar evolutionary theory, giving, of course, only a lower limit to the age of the Universe. On page 127 of this issue, Harvey Butcher provides a new calibration of the stellar evolutionary age scale, by comparison with radioactive dating of stars near the Sun, that suggests a large systematic error in the current models. If correct, both the accepted physics of stellar evolution and the age of the Universe require substantial revision.

The technique of age dating using radioactive elements was devised by Rutherford (Nature 123, 313-314; 1929), and is now a well-known technique. The principle is straightforward: radioactive parent atoms decay into one or more daughter elements at a rate controlled by a measurable constant, the half life. Thus if one counts the relative number of parent and daughter elements, and knows the half life, it is easy to deduce the time at which the pure parent element was created. In practice, for astrophysical dating it is also necessary to know the initial (relative) abundance of parent and daughter elements. As heavy elements are continually created from lighter ones (by stellar nuclear burning) throughout the lifetime of the Galaxy, it is also necessary, if comparing old and new stars, to allow for the rate of creation. Because of these problems, as well as the observational difficulty in measuring spectral features from rare elements, considerable uncertainties remained in previous analyses, and the derived age of the Universe fell in the range 10-25 gigayears (Gyr).

The most useful radioactive pair for cosmology so far has been ${ }^{232} \mathrm{Th} /{ }^{238} \mathrm{U}$, as the half life for ${ }^{232} \mathrm{Th}$ is $20 \mathrm{Gyr}$, similar to the time of interest. Rather than measure the ratio of these elements, Butcher has compared the elemental abundance of ${ }^{232}$ Th directly with that of a stable element, neodymium (mostly ${ }^{142} \mathrm{Nd}$ ), which is relevant as its spectral line of interest is excited in the same part of the stellar atmosphere as that of thorium. Thus, effects specific to the atmospheres of individual stars are minimized.

The price paid, however, is that thorium and neodymium are created in different astrophysical processes, so that the time dependence of their relative abundance becomes problematic. Thorium is created in the r-process, involving the rapid absorption of neutrons in a very high neutron-density environment. This process is poorly understood, but probably occurs mostly in rapid helium burning in supernovae and novae. Neodymium is formed mostly by s-process, involving the slow absorption of neutrons in a lower neutron-density environment. This occurs mostly in the normal late evolution of intermediate-mass stars. A second timedependent source of thorium is, of course,

Plans to launch space sculptures into Earth orbit to celebrate the centenary of the Eiffel Tower have caused great alarm among astronomers - see for example the recent News and Views article of Paul Murdin (Nature 326, 125; 1987). But even now, the purely functional satellites that populate the heavens are interfering with observations, as highlighted by a recent letter in The Astrophysical Journal (317, L39-L44; 1987) from Paul D. Maley of the Rockwell Shuttle Operations Company. The illustration, which comes from that paper, emphasizes dramatically how crowded space has become. It shows a computer-generated 'snapshot' of all the catalogued satellites at 00.00 hours Universal Time on 1 January 1987.

In particular, Maley considers flashes of light recorded photographically and also observed by eye in the vicinity of the constellations Perseus and Aries (MacRobert, A. Sky Telesc. 70, 54; 1985: Katz, B. et al. Astrophys. J. 317, L33L37; 1986). It was considered

that these flashes, lasting about 1 second, might be caused by flare stars, or $\gamma$-ray or $\mathrm{X}$-ray bursters. For this reason there was interest in the magnitude (intensity), spectra and light curves (time variation of intensity) of the flashes. But Katz et al. showed that the flashes were too frequent to be caused by such sources or by meteors.

In his analysis, Maley investigated the probability that the events were caused by reflections of the sun off satellites. For example, for one event on 19 March 1985 , he traced the trajectories of 5500 objects, the decay of its parent nuclei. Each of these sources will occur with a natural timescale which is the age of our Galaxy, the Milky Way, and so needs careful consideration when using a sample of stars in the Milky Way disk, as Butcher has done.

At face value, Butcher's excellent data show no decay at all in the $\mathrm{Th} / \mathrm{Nd}$ relative abundance. This implies a short age scale, with the oldest stars being no more than 12 Gyr, rather than $18 \mathrm{Gyr}$, old. The absence of any change in the ratio is surprising, and suggests that careful consideration of the relative production histories of $\mathrm{r}$ - and $\mathrm{s}$ process elements is necessary. If the conclusion is correct, however, the implications are profound. Not only is the currently accepted physics of stellar evolution incomplete, but the Universe may be substantially younger than presently believed. Extension of this experiment to elements with different production histories and half lives is eagerly awaited.

Gerry Gilmore is at the Institute of Astronomy, Cambridge University, Madingley Road, Cambridge $C B 3$ OHE, $U K$.

of which some 1400 were above the local horizon within the hour at which the observation was made. There are two possible candidates to be the source of the flashes, the most likely being Cosmos 1400 . Telescopic observation of specular reflections of sunlight of Cosmos 1400 confirmed Maley's suspicion. Maley could similarly account for three other flashes that he chose to investigate. A further three flashes, from a separate observation

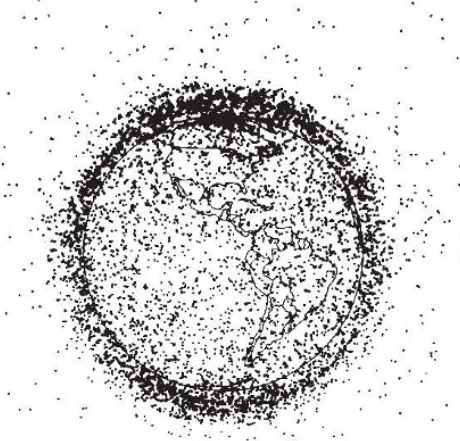

(Pedersen, H. et al. Nature 312, 46-48; 1984) remain unexplained and could originate from the supernova remnant $\mathrm{N} 49$, as originally postulated.

There is burgeoning interest in studies of short-duration phenomena such as flare stars, but the investigations are in their infancy, requiring large-angle, highresolution detectors to identify these infrequent events. As the skies become more cluttered, it seems that astronomers' patience will be increasingly tested - what price space sculptures? 